\title{
Oral Presentations at the 10th Canadian Conference on Dementia (CCD) Québec City, October 3, 2019
}

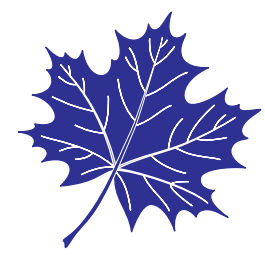

DOI:https://doi.org/10.5770/cgj.23.410

Correlation Between Amyloid Reduction and Clinical Outcomes: Exploratory Analyses from the Gantenerumab Scarlet Road and Marguerite Road Open-Label Extension Studies

Paul Delmar ${ }^{1}$, Geoffrey A Kerchner ${ }^{1}$, Guoqiao Wang², Randall Bateman $^{2}$, Gregory Klein ${ }^{3}$, Mirjana Andjelkovic ${ }^{1}$, Danielle Abi-Saab ${ }^{4}$, Szofia Bullain 4 , Alonso Montoya ${ }^{5}$, Paulo Fontoura ${ }^{4}$, Rachelle Doody 6

${ }^{1}$ Roche Pharma Research and Early Development; ${ }^{2}$ Washington University, School of Medicine, St. Louis, MO, USA; ${ }^{3}$ Roche/Genentech Product Development, Neuroscience, Basel, Switzerland; ${ }^{4}$ Roche Pharma Research and Early Development, Basel, Switzerland; '5edical Strategy, Hoffmann-La Roche, Mississauga, ON; ${ }^{6}$ Genentech Inc., South San Francisco, CA, USA

Background/Objectives: Gantenerumab, a fully human monoclonal antibody, is under investigation as a diseasemodifying treatment for Alzheimer's disease (AD). In the ongoing SCarlet RoAD (SR [NCT01224106]) and Marguerite RoAD (MR [NCT02051608]) open-label extension (OLE) studies, patients with $\mathrm{AD}$ who received gantenerumab uptitrated to subcutaneous doses $\leq 1200 \mathrm{mg}$ monthly for 2 years exhibited high levels of PET amyloid reduction, such that 52\% of patients improved below the amyloid positivity threshold.(1) Here we explore the relationship between amyloid reduction and clinical outcomes during the MR and SR OLE studies

Methods: Amyloid PET reductions were quantified using a prespecified $\operatorname{method}^{(2)}$ and centiloid transformation. ${ }^{(3)}$ Clinical outcomes evaluated included CDR-SB, ADAS-COG11, and MMSE. The analysis included all currently available patients with week 104 PET scans from MR and SR OLE studies. Exploratory analyses included comparing clinical endpoint trajectories in patients with higher versus lower levels of PET amyloid reductions based on median split using MMRM (adjusted for baseline clinical endpoint value), and fitting a newly developed joint model for the simultaneous evaluation of biomarker and clinical endpoint longitudinal data. ${ }^{(4)}$

Results: Large mean amyloid reductions ( -38 centiloids [SD 38.1] at week $52[\mathrm{~N}=67]$ and -59 centiloids [SD 35.3] at week $104[\mathrm{~N}=39]$ ), often to below the amyloid positivity threshold, were observed. At week 104, point estimates indicated $17-46 \%$ less clinical decline in the subgroup with larger ( $>$ median) PET reductions versus those with smaller reductions. Similar directional trends were observed across all analysis methods and clinical endpoints

Conclusion: Larger amyloid reductions at week 104 were associated with a trend toward less decline in clinical scales. Given the limited sample size and absence of a placebo arm, clinical outcome data should be interpreted with caution. However these data, together with the favorable safety profile observed at these dose levels, support the rationale for further investigation of the clinical efficacy of gantenerumab and 2 ongoing pivotal PhIII trials in patients with early (prodromalto-mild) AD (GRADUATE I [NCT03444870]; GRADUATE II [NCT03443973]). 1.Klein, et al. CTAD 2018 2.Ostrowitzki S, et al. Alzheimers Res Ther 2017 3.Klunk WE, et al. Alzheimers Dem 2015 4.Wang G,et al. AlzheimersDem2018

Towards Dementia Friendly Emergency Departments: A Mixed Method Exploratory Study Identifying Opportunities to Improve the Quality and Safety of Care for People with Dementia in Emergency Departments

Courtney Shaw ${ }^{1}$, Gerrard Armitage ${ }^{2}$, Andrea Capstick ${ }^{3}$

${ }^{1}$ SE Research Centre; 'University of Bradford/Bradford District Care Trust; ${ }^{3}$ University of Bradford

Background/Objectives: People living with dementia (PLWD) are frequent users of Emergency Departments (ED's). They tend to have complex care needs as a result of multi-morbidities, cognitive impairments and enhanced need for social support. Despite the prevalence of dementia in ED patients, the physical environment and care processes are typically not designed to meet their holistic needs. The objective of this project was to create a model of dementia friendly emergency care

Methods: This is a mixed and multiple methods study which used an iterative and sequential design to present a holistic evaluation of the current experiences of the key parties - patients, caregivers, and ED staff involved in receiving and providing 
care. These methods included; 1) A literature review on geriatric and dementia friendly ED's 2) A national survey $(\mathrm{N}=403)$ ) to explore current experiences of ED care from the perspective of patients and caregivers; 3) 32 hours of observation in ED and qualitative interviews with health professionals $(\mathrm{N}=29)$ to understand the barriers and facilitators of providing dementia friendly care in ED. The theoretical perspective of the Human Factors Approach to patient safety and systems engineering underpins this work. The project included PWD and caregivers as collaborators and co-designers in both the development of the research tools and in shaping the project outputs.

Results: A set of statements on the key components of Dementia Friendly ED's was co-designed by PLWD and caregivers from results. These statements highlight the importance of empathy, Dementia awareness, and a dyad approach to care. Staff interviews highlight barriers which may affect the healthcare team's ability to provide effective dementia careincluding poor integration of communication systems which impacts quality and continuity of care, physical environments which cause PWD distress, and difficulties with staff recruitment, retention and training. These systemic challenges both give rise to and exacerbate poor organisational and safety cultures which can lead to staff-patient interactions become less relationship-centred. These outputs are used to create a model which highlights practical changes to the physical environment of the ED, the care processes required to provide holistic care, and the staffing complement required. The model's is underpinned by, and predicated on, organisational culture which values relationship centered care.

Conclusion: The complex needs of PWD are fundamentally misaligned with the rapid triage systems and intervention based focus of a typical ED. This misalignment of patient needs and the current model of service provision leads to poor patient and caregiver outcomes (anxiety, overstimulation, unmet needs) and poor staff experiences (feeling overworked, unsupported and burnt out). Adopting some, or all of the proposed adaptations in structures, care processes, and approaches to care identified in this research is likely to help align ED care to priorities of PLWD and caregivers.

\section{Canadian Indigenous Cognitive Assessment (CICA): Inter-Rater Reliability and Criterion Validity Using a Clinical Sample}

Jennifer Walker ${ }^{1}$, Wayne Warry ${ }^{2}$, Melissa Blind ${ }^{2}$, Christopher Patterson ${ }^{3}$, Cheryl Allaby ${ }^{3}$, Karen Pitawanakwat ${ }^{4}$, Yantao Zhao', Andrine Lemieux ${ }^{2}$, Kristen Jacklin ${ }^{2}$, Megan E O'Connell ${ }^{5}$

${ }^{1}$ School of Rural and Northern Health, Laurentian University; 'University of Minnesota Medical School Duluth, Memory Keepers Medical Discovery Team; ${ }^{3}$ Department of Medicine, McMaster University; ${ }^{4}$ Nahndawehtchigeh Gamig Wiikwemkoong Health Center; ${ }^{5}$ Department of Psychology, University of Saskatchewan
Background/Objectives: Dementia is a growing concern for Indigenous people worldwide. Age and other factors including high rates of multiple, complex health conditions at a younger age of onset and a combination of social, historical and colonial factors have led to projections of increased dementia in First Nations populations. Yet early detection of dementia in Indigenous populations remains challenging as current cognitive assessment tools are shown to be less reliable when used in these populations. The need for culturally appropriate clinical measures for dementia was identified by Indigenous community members and health care providers in Ontario, Saskatchewan and Alberta as a priority.

Methods: The Canadian Indigenous Cognitive Assessment (CICA) tool is a culturally-informed cognitive assessment tool that was adapted by Anishinaabe First Nations communities on Manitoulin Island, Ontario. The CICA was validated over a period of several years using a multi-phase approach that included adaptation, translation, piloting, reliability and validity testing. The CICA was successfully validated in summer 2018 and takes approximately 15 minutes to complete, assesses 11 domains of cognition and provides a final score out of a possible 39 points. This study reports the inter-rater reliability and criterion validity of the newly developed CICA.

Results: The CICA demonstrated strong reliability $(\mathrm{ICC}=$ $0.95(0.85,0.98))$ and validity. The ideal cut-point to identify likely cases of dementia was a score of less than or equal to 34 , for which the sensitivity was $100 \%$, specificity was $85 \%$, likelihood ratio plus (LR+) was 6.5 and the AUC was 0.98 (95\% CI: 0.94 to 1.00). The results indicated a range of domain discriminability (Partial R-square range $=0.04-0.57$ ). Two cognitive domains alone accounted for $71 \%$ of the variance in dementia status.

Conclusion: The CICA is the first tool of its kind in Canada. The successful validation of the CICA tool on Manitoulin Island marks a significant step forward in the broader effort to provide culturally-safe health care services for Indigenous populations. The implications of the CICA for improved detection of cognitive impairment and dementia among Indigenous populations, culturally-safe pathways to formal healthcare, dementia surveillance, resource allocation and policy and planning are substantial.

Can a Group Exergame Intervention Impact Balance, Movement Confidence, and Cognitive Function in People with Dementia or Mild Cognitive Impairment?

Erica Dove ${ }^{1}$, Arlene Astel1 ${ }^{2}$

${ }^{1}$ University of Toronto; 2 Toronto Rehabilitation Institute

Background/Objectives: Participation in exercise programs can benefit people with cognitive impairment (PCI; e.g. dementia). However, many exercise programs offered to 


\section{CCD ORAL PRESENTATIONS}

this population are passive, unengaging, and repetitive, resulting in poor engagement and long-term adherence. The potential of integrating video games and exercise programs (i.e. 'exergame' programs) is being explored to encourage exercise participation among PCI. However, the impacts on key variables including balance, movement confidence, and cognitive function have yet to be determined. This study aims to examine the impacts of a group exergame intervention (Xbox Kinect bowling) for PCI on balance, movement confidence, and cognitive function.

Methods: This within-participants design includes measurement at pre- and post-intervention. Twenty-four PCI are being recruited from two adult day programs in Durham Region, ON. At pre-test, participants will complete a demographic survey, the Mini Balance Evaluation Systems Test (MiniBEST), and the Montreal Cognitive Assessment (MoCA). Participants will play an Xbox Kinect bowling game in a group setting, twice per week for ten weeks (20 sessions). Participants will be video recorded at three time points (start, middle, and end) to capture physical indicators of movement confidence (e.g. fluency of motion, visual focus, hesitation) during the intervention. At post-test, the Mini-BEST and MoCA will be repeated.

Results: Quantitative data collected through the Mini-BEST, coded video recordings, and the MoCA will be compared from pre- to post-test using paired t-tests. An ANCOVA with post hoc analyses will also be performed to account for covariates (e.g. number of intervention sessions attended).

Conclusion: The exergame intervention has the potential to positively impact participants' physical function, specifically balance (score on the Mini-BEST) and movement confidence (coded from video recordings). This will confirm the feasibility and potential benefits of using MBT to deliver video gamebased exercise interventions to PCI. There is also potential for the MBT intervention to positively impact cognitive function of PCI (as measured through MoCA score). This work can be used as the basis for developing both specific software and future video game-based exercise programs for PCI.

Use of Medium Chain Triglyceride (MCT) Oil in Subjects with Alzheimer's Disease: A Randomized Double-Blind Placebo Cross Over Study, with an Open Label Extension

Angela Juby ${ }^{1}$, Diana Mager ${ }^{1}$, Christopher Davis ${ }^{1}$, David Jay ${ }^{2}$, Toni Blackburn ${ }^{3}$

${ }^{1}$ University of Alberta; ${ }^{2}$ Grant MacEwan University; ${ }^{3}$ Alberta Health S122ervices

Background/Objectives: Cerebral glucose metabolism is impaired in subjects with Alzheimer's disease (AD). Under conditions of low glucose availability the brain has the cap- acity to use ketones as an alternative energy source. The usual source of ketones is break down of stored triglycerides, and occurs in fasting or low calorie intake states. This endogenous ketone production has been shown to be helpful in other neurological diseases, such as epilepsy, but not in AD. Practically, it is difficult to reduce calories sufficiently for an endogenous ketogenic response. Medium chain triglycerides (MCT) are known to be an exogenous (dietary) source of ketones.

Methods: This study is to evaluate the impact, if any, of MCT oil supplementation in AD subjects. It is a six month randomised, double blind, placebo, cross over study, with 6 month open label extension evaluating MCT oil versus olive oil on cognition, behavior and activities of daily living function in community dwelling $\mathrm{AD}$ subjects. One month dose titration $(15 \mathrm{mls}-30 \mathrm{mls}-45 \mathrm{mls}$ or maximum tolerated dose (MTD) daily) followed by 3 months of MTD, occurred in the cross-over phase. This was followed by 6 months of open label extension of MTD MCT oil. Subjects were allowed to continue all medications including cholinesterase inhibitors, mematine and antidepressants provided the doses remained stable during the study. This study was approved by the Health Canada (HC) and the local Ethics Board. Patients with concomitant Diabetes were not included as per $\mathrm{HC}$ requirement.

Results: Twenty AD subjects completed the first 6 months, and 19 completed the full 15 months. Participant's age ranged from 54-84yrs (average age of 72.6yrs), and included 11 men and 9 women. 70\% had College/University education. Baseline Mini Mental status Examination (MMSE) score 22.6/30 (10-29). Montreal Cognitive Assessment (MoCA) 15.6/30 (4-30). Baseline Cognigram ${ }^{\circledR}$ Part 1 ranged from 65-106, Part 2 from 48-107. There were no significant differences between the two groups at baseline. Apo E4 status was homozygous in one subject, and heterozygous in nine $(50 \%$ overall positive ApoE4). Average MCT oil consumption was 1.8 tablespoons daily $(25.2 \mathrm{~g}, 234 \mathrm{kcal})$. There was individual variability in MMSE and MoCA over the course of the study, but most remained stable or improved. Four (20\%) declined more rapidly than expected (based on their initial MMSE). There was no difference in average MCT intake in "decliners". No change in serum lipids or $\%$ body fat occurred.

Conclusion: Only recently has attention been drawn to the use of MCT oil as a source of ketones for AD subjects, with the hope that this may improve their brain metabolism and thereby cognitive function. The objective of this study is to evaluate the impact, if any, of MCT oil on cognition, activities of daily living (ADLs) and behavior in AD subjects. Preliminary analysis shows stabilization in cognition. This is not what would have been expected in these established AD patients. The differences between responders and decliners is being evaluated further. 